\title{
ON ODD DIMENSIONAL SURGERY WITH FINITE FUNDAMENTAL GROUP
}

\author{
JEAN-CLAUDE HAUSMANN ${ }^{1}$
}

\begin{abstract}
One proves that, for any finite group $G$ and homomorphism $\omega: G \rightarrow \mathbf{Z} / 2 \mathbf{Z}$, the natural homomorphism $L_{2 k+1}^{h}(\mathbf{Z} G, \omega) \rightarrow L_{2 k+1}^{h}(\mathbf{Q} G, \omega)$ between Wall surgery groups is identically zero. Some results concerning the exponent of $L_{2 k+1}^{h}(\mathrm{Z} G ; \omega)$ are deduced.
\end{abstract}

1. Introduction. Let $G$ be a group and $\omega: G \rightarrow \mathbf{Z} / 2 \mathbf{Z}$ be a homomorphism (orientation character). Let $L_{n}^{h}(G ; \omega)$ be the Wall surgery obstruction group in dimension $n$ for surgery to a homotopy equivalence. By changing $\mathbf{Z} G$ into $\mathbf{Q} G$ in the definition of $L_{n}^{h}$, one gets groups $L_{n}^{h}(Q G ; \omega)$. They contain the obstruction for surgery to an $[n-1] / 2$-connected rational homology equivalence. There is a natural homomorphism $r: L_{n}^{h}(G ; \omega) \rightarrow L_{n}^{h}(\mathbf{Q} G ; \omega)$ which is used in the long exact localization sequence for surgery obstruction groups of W. Pardon [P1].

Our first result is the following:

THEOREM 1. For any finite group $G$ and homomorphism $\omega: G \rightarrow \mathbf{Z} / 2 \mathbf{Z}$, the homomorphism $r: L_{2 k+1}^{h}(G ; \omega) \rightarrow L_{2 k+1}^{h}(\mathbf{Q} G ; \omega)$ is the zero homomorphism.

Consequently, in the odd dimensional case with a finite fundamental group, the surgery to a rational homotopy equivalence is always possible. The obstruction for surgery to a homotopy equivalence is always expressible by the linking numbers approach developed in $[\mathbf{K M}],[\mathbf{W 1}]$ and $[\mathbf{C}]$. Since the kernel of $r$ is annihilated by $8[\mathbf{C}]$, one has

COROLlaRY 2. For any finite group $G$ and homomorphism $\omega: G \rightarrow \mathbf{Z} / 2 \mathbf{Z}$, $L_{2 k+1}^{h}(G ; \omega)$ is annihilated by 8 .

It has been conjectured that this exponent is 4 , at least for a large class of finite groups. In this direction, a slight improvement of the proof of Theorem 1 gives the following results:

THEOREM 3. Let $1 \rightarrow N \rightarrow G \rightarrow^{\Phi} B \rightarrow 1$ be an exact sequence of finite groups, and let $\omega: B \rightarrow \mathbf{Z} / 2 \mathbf{Z}$ be a homomorphism. Suppose that $N$ is a 2-group. If $y \in \operatorname{Ker}\left(\Phi_{*}: L_{2 k+1}^{h}(G ; \Phi \circ \omega) \rightarrow L_{2 k+1}^{h}(B ; \omega)\right)$, then $4 y=0$.

Received by the editors May 13, 1976 and, in revised form, July 30, 1976. AMS (MOS) subject classifications (1970). Primary 18F25, 57D65.

1 Supported in part by NSF Grant MPS72-05055 A03. 
COROllaRY 4. For any finite 2-group $G$ and any homomorphism $\omega: G$ $\rightarrow \mathbf{Z} / 2 \mathbf{Z}, L_{2 k+1}^{h}(G ; \omega)$ is annihilated by 4 .

Corollary 5. Let $G$ be a finite group whose 2-Sylow subgroup is normal. Suppose that $\omega$ is trivial (the orientable case). Then $L_{2 k+1}^{h}(G ; \omega)$ is annihilated by 4.

For instance, the assumptions of Corollary 5 are fulfilled if $G$ is a finite nilpotent group, since a finite nilpotent group is the product of its Sylow subgroups $[\mathbf{H}]$.

A part of these statements is more or less known to be obtainable by different methods. Some particular cases are also deducible from published results of other authors. For instance, if $k$ is odd, Theorem 1 is obvious since $L_{2 k+1}^{h}(\mathbf{Q} G ; \omega)=0[\mathbf{C}]$. On the other hand, in the orientable case ( $\omega$ trivial), Corollary 2 can be deduced from [W3, exact sequence, p. 78 and remark (4), p. 2]. Corollaries 4 and 5 are deducible from [W3] when $G$ is abelian. Recently, W. Pardon independently found an algebraic proof of Corollary 4 and A. Bak computed $L_{n}^{h}(G ; \omega)$ when $G$ has its 2-Sylow subgroup normal and abelian [Bak2]. Finally, an announcement of Theorem 1 when $G$ is abelian was published by R. M. Geist [G]. Our proofs are independent from these results and our approach is quite different.

In $\S 2$, we give a sufficient condition for a degree one map between a manifold pair and a Poincaré pair (of odd dimension) to be a rational homotopy equivalence. In $\S 3$, we establish a functoriality property for a part of the localization exact sequence of surgery groups ([P1] and [P2]). It would be interesting to know in which generality such a functoriality property holds.

Results of $\$ \S 2$ and 3 are used in $\$ 4$ for proving Theorem 1; finally, Theorem 4 and Corollaries 4 and 5 are proved in $\$ 5$.

I am grateful to W. Pardon for conversations, to C.T.C. Wall and A. Bak for commenting on these results, and to the referee for a great simplification of my original proof of Proposition 2.1.

2. A class of rational homology equivalences. Denote, as usual, by $\mathbf{Z}_{(p)}$ the subring of $\mathbf{Q}$ of fractions expressible with a denominator prime to $p$. This section is devoted to proving the following proposition:

Proposition 2.1. Let $(X, Y)$ be a Poincaré pair of dimension $2 k+1$ such that $\pi_{1}(Y) \simeq \pi_{1}(X)$ is a finite group $G$. Let $N \subset G$ be a normal p-subgroup of $G(p$ some prime). Consider a map $f:(M ; \partial M) \rightarrow(X ; Y)$ of degree one $\left(M^{2 k+1} a\right.$ compact manifold), with $f \mid \partial M$ a homotopy equivalence. Suppose that $f$ is $k$ connected and is a $\mathbf{Z}(G / N)$-homology equivalence (local coefficients). Then $f$ is a $\mathbf{Z}_{(p)^{-}}$-homology equivalence (and thus a rational homology equivalence).

Proof. If $B$ is a $\mathbf{Z} G$-module, we denote by $K_{k}(M ; B)$ the $\mathbf{Z} G$-module $H_{k+1}(f ; B)$ and $K_{k}(M)$ is used for $K_{k}(M ; Z G)$. By [CS, Lemma 1.4] one has $K_{k}(M ; \mathbf{Z}(G / N))=K_{k}(M) \otimes_{\mathbf{Z} G} \mathbf{Z}(G / N)$. This last module is $\mathbf{Z}$-isomorphic to $K_{k}(M) \otimes_{\mathbf{Z} N} \mathbf{Z}=K_{k}(M) / I \cdot K_{k}(M)$, where $I$ is the augmentation ideal of $N$. 
Since $f$ is a $\mathbf{Z}(G / N)$-homology equivalence, one has $K_{k}(M ; \mathbf{Z}(G / N))=0$, and then $K_{k}(M)=I \cdot K_{k}(M)$.

Since $K_{k}(M)$ is finitely generated [W2, Lemma 2.3] and $G$ is finite, $K_{k}(M) / p K_{k}(M)$ is a finite abelian $p$-group. The action of the finite $p$-group $N$ on any finite abelian $p$-group $L$ is nilpotent, i.e. $I^{s} \cdot L=0$ for $s$ large enough. (The proof goes like in [H, pp. 47 and 155].) Therefore $K_{k}(M)=p K_{k}(M)$, which is equivalent to $K_{k}(M) \otimes_{\mathbf{z}} \mathbf{Z}_{(p)}=0$. But $K_{k}(M) \otimes_{\mathbf{Z}} \mathbf{Z}_{(p)}=K_{k}(M)$ $\otimes_{\mathbf{Z G}} \mathbf{Z}_{(p)} G=K_{k}\left(M ; \mathbf{Z}_{(p)} G\right)$, the last isomorphism by [CS, Lemma 1.4]. Thus, $K_{k}\left(M ; \mathbf{Z}_{(p)} G\right)=0$ and $f$ is a $\mathbf{Z}_{(p)}$-homology equivalence.

3. Partial functoriality for the surgery group localization exact sequence. We will use a functorial property of the following leg of the Pardon exact sequence [P1]:

$$
\begin{aligned}
L_{2 k+2}^{h}(\mathbf{Q} G, \omega) & \rightarrow L_{2 k+1}^{t}(\mathbf{Z} G ; \mathbf{Z}-\{0\}) \\
& \rightarrow L_{2 k+1}^{h}(G, \omega) \rightarrow L_{2 k+1}^{h}(\mathbf{Q} G, \omega) .
\end{aligned}
$$

This functoriality property is implied by the corresponding property for this other formulation of the sequence [P2, Theorem 2.1]:

$$
W_{0}^{-\lambda}(\mathbf{Q} G) \rightarrow W_{0}^{-\lambda}(\mathbf{Q} G / \mathbf{Z} G) \rightarrow W_{1}^{\lambda}(\mathbf{Z} G) \rightarrow W_{1}^{\lambda}(\mathbf{Q} G)
$$

where $\lambda=(-1)^{k}$. (See [P2] for the definitions.) Sequences (3.1) and (3.2) are related by the following commutative diagram:

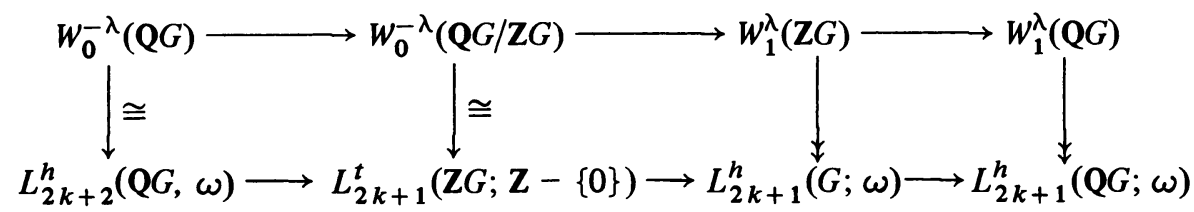

where the left two vertical arrows are identity maps (the groups are identical) and two right vertical arrows divide out by $w_{1}^{\lambda}=\left(\begin{array}{ll}0 & 1 \\ \lambda & 0\end{array}\right)$.

Let $\mathscr{F}$ be the category whose objects are pairs $(G ; \omega)$, where $G$ is a finite group and $\omega: G \rightarrow \mathbf{Z} / 2 \mathbf{Z}$ is a homomorphism. A morphism $F:\left(G, \omega_{G}\right)$ $\rightarrow\left(H, \omega_{H}\right)$ of $\mathscr{F}$ is a homorphism $f: G \rightarrow H$ such that $\omega_{G}=\omega_{H} \circ f$. Let $A b$ denote the category of abelian groups. The group rings $R G(R=\mathbf{Z}$ or $\mathbf{Q})$ are understood to be endowed with the involution $\overline{\sum n_{g} g}=\sum n_{g} \omega(g) g^{-1}$.

Proposition 3.4. The correspondences

$$
(G, \omega) \rightarrow W_{i}^{\lambda}(R G), \quad i=0 \text { or } 1, \lambda= \pm 1,
$$

and 


$$
(G, \omega) \mapsto W_{0}^{\lambda}(\mathbf{Q} G / \mathbf{Z} G)
$$

give rise to functors from $\mathscr{F}$ to $A b$ so that sequence (3.2) (and thus (3.1)) is functorial.

Proof. $W_{1}^{\lambda}(R G)$ are functors in the usual way. The only nonobvious point is to define $f_{*}: W_{0}^{\lambda}(\mathbf{Q} G / \mathbf{Z} G) \rightarrow W_{0}^{\lambda}(\mathbf{Q} H / \mathbf{Z} H)$ for an $\mathscr{F}$-morphism $f:\left(G, \omega_{G}\right)$ $\rightarrow\left(H, \omega_{H}\right)$. By definition of $W_{0}^{\lambda}(\mathbf{Q} G / \mathbf{Z} G)\left[\mathbf{P 2}\right.$, p. 10] it suffices to have $f_{*}$ defined on classes in $W_{0}^{\lambda}$ which are represented by a triple $(M, \varphi, \psi)$, where

(1) $M$ is a finite $\mathbf{Z} G$-module admitting a short free $\mathbf{Z} G$-resolution

$$
0 \rightarrow F_{1} \stackrel{\mu}{\rightarrow} F_{0} \rightarrow M \rightarrow 0
$$

(2) $\varphi: M \times M \rightarrow \mathbf{Q} G / \mathbf{Z} G$ is a nonsingular $\lambda$-hermitian form.

(3) $\psi \cdot M \rightarrow \mathbf{Q} G / S_{\lambda}(\mathrm{ZG})$ is a function satisfying (i)-(iii) of [P2].

Let us define the image by $f_{*}$ of a class $(M, \varphi, \psi)$ to be the class of $\left(M^{\prime}, \varphi^{\prime}, \psi^{\prime}\right)$, where

(1) $M^{\prime}=M \otimes_{\mathrm{ZG}} \mathbf{Z} H$.

(2) $\varphi^{\prime}: M^{\prime} \times M^{\prime} \rightarrow \mathbf{Q} H / \mathbf{Z} H$ is defined by $\varphi^{\prime}(x \otimes a, y \otimes b)=\bar{a} f(\varphi(x, y)) b$ (see [Ba, §6]).

(3) $\psi^{\prime}: M^{\prime} \rightarrow \mathbf{Q} H / S_{\lambda}(\mathbf{Z} H)$ is the unique extension of $\psi$ such that $\psi^{\prime}(x \otimes 1)$ $=f(\psi(x))[\mathbf{B a}, 6.3]$.

Let us check that $\left(M^{\prime}, \varphi^{\prime}, \psi^{\prime}\right)$ actually determines a class in $W_{0}^{\lambda}(\mathbf{Q} H / \mathbf{Z} H)$. $\boldsymbol{M}^{\prime}$ is a finite $\mathbf{Z} H$-module; it admits the following short free resolution:

$$
0 \rightarrow F_{1} \otimes \mathbf{Z} H \stackrel{\mu \otimes 1}{\longrightarrow} F_{0} \otimes \mathbf{Z} H \rightarrow M^{\prime} \rightarrow 0
$$

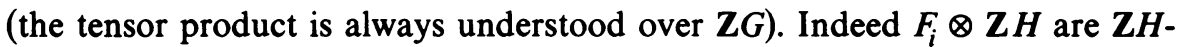
free of same $\mathrm{Z} H$-rank. Since $H$ is a finite group, $F_{1} \otimes \mathbf{Z} H$ and $F_{2} \otimes \mathbf{Z} H$ have same finite $\mathbf{Z}$-rank. Thus $\mu \otimes 1$ is injective.

To prove that $\varphi^{\prime}$ is nonsingular, it suffices [Ba, p. 45] to check that the homomorphism

$$
j_{M}: \operatorname{Hom}_{\mathbf{Z} G}(M ; \mathbf{Q} G / \mathbf{Z} G) \otimes \mathbf{Z} H \rightarrow \operatorname{Hom}_{\mathbf{Z} H}\left(M^{\prime} ; \mathbf{Q} H / \mathbf{Z} H\right)
$$

given by $j_{M}(h \otimes a)(x \otimes b)=\bar{a} f(h(x)) b$ is an isomorphism (observe that $\mathbf{Q} G / \mathbf{Z} G \otimes \mathbf{Z} H \simeq \mathbf{Q} H / \mathbf{Z} H)$. By [P2, Proposition 1.4], $\operatorname{Hom}_{\mathbf{Z G}}(M ; \mathbf{Q} G / \mathbf{Z} G)$ has the following free resolution:

$$
0 \rightarrow \operatorname{Hom}_{\mathbf{Z} G}\left(F_{0} ; \mathbf{Z G}\right) \stackrel{\bar{\mu}}{\rightarrow} \operatorname{Hom}_{\mathbf{Z} G}\left(F_{1} ; \mathbf{Z G}\right) \rightarrow \operatorname{Hom}_{\mathbf{Z} G}(M ; \mathbf{Q} G / \mathbf{Z G}) \rightarrow 0 .
$$

As above, tensoring by $\mathbf{Z} H$ leaves this sequence exact. Hence, one has 


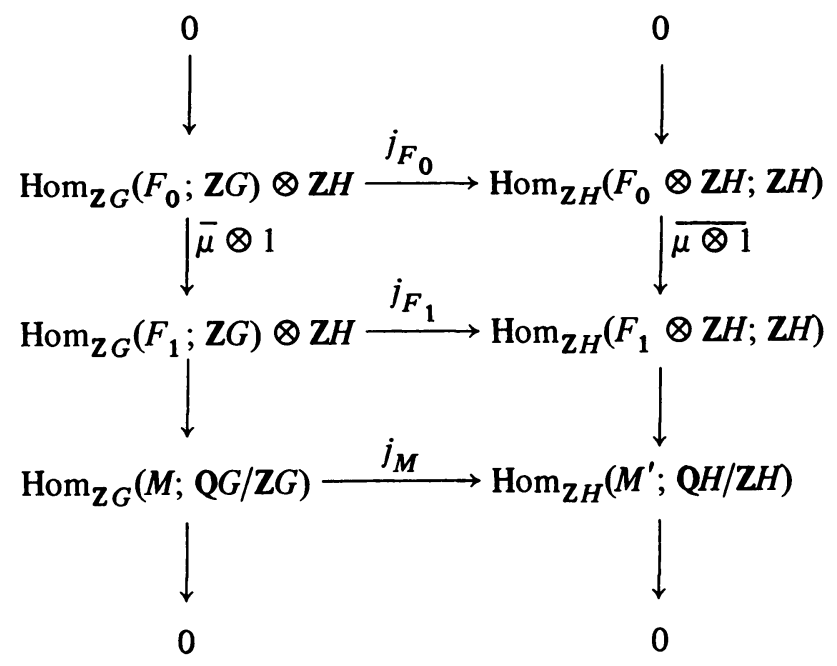

The commutativity of the lowest square comes from the definitions of $j_{F_{1}}$ and $j_{M}$ and of the identification of coker $\bar{\mu}$ with $\operatorname{Hom}_{\mathbf{Z G}}(M ; \mathbf{Q} G / \mathbf{Z G})[\mathbf{P 2},(1.5)]$. Clearly, $j_{\mathbf{Z} G}$ is an isomorphism. By additivity, $j_{F_{0}}$ and $j_{F_{1}}$ are isomorphisms. Thus, $j_{M}$ is an isomorphism.

Finally, one checks without difficulty that a kernel is mapped onto a kernel. Thus $f_{*}: W_{0}^{\lambda}(\mathbf{Q} G / \mathbf{Z} G) \rightarrow W_{0}^{\lambda}(\mathbf{Q} H / \mathbf{Z} H)$ is well defined.

The functoriality of sequence (3.2) then comes directly from the definitions of each arrow.

4. Proof of Theorem 1. Theorem 1 will be proven first when $G$ is a finite 2group, then when $G$ is a 2-hyperelementary group and last in the general case using the induction theorem due to Dress. We shall make use of the following lemma, in which $r: L_{2 k+1}^{h}(G ; \Phi \circ \omega) \rightarrow L_{2 k+1}^{h}(\mathbf{Q} G ; \Phi \circ \omega)$ is the natural homomorphism, as in the Introduction.

LEMMA 4.1. Let $1 \rightarrow N \rightarrow G \rightarrow^{\Phi} B \rightarrow 1$ be an extension of finite groups, with $N$ a p-group. Let $\omega: B \rightarrow \mathbf{Z} / 2 \mathrm{Z}$ be a homomorphism. Then, for all $x$ $\in \operatorname{Ker}\left(L_{2 k+1}^{h}(G ; \omega \circ \Phi) \rightarrow L_{2 k+1}^{h}(B ; \omega)\right)$, one has $r(x)=0$.

Proof. Let $M^{2 k}$ be a manifold, $k \geqslant 3$, with $\pi_{1}(M)=G$ and orientation homomorphism $\omega \circ \Phi$. Accordingly [W2, Theorem 6.5] there exists a cobor$\operatorname{dism}\left(W^{2 k+1}, M, M^{\prime}\right)$ and a normal map of degree one $(f, b) f:\left(W, M, M^{\prime}\right)$ $\rightarrow(M \times I, M \times\{0\}, M \times\{1\})$ such that $f \mid M=\mathrm{id}_{M}$, the map $f \mid M^{\prime}$ is a homotopy equivalence, and the surgery obstruction $\theta(f, b) \in L_{2 k+1}(G ; \omega \circ \Phi)$ is equal to $x$.

Let $\Gamma_{2 k+1}^{h}\left(\mathbf{Z} G \rightarrow^{\Phi} \mathbf{Z} B\right)$ be the Cappell-Shaneson surgery obstruction group [CS]. This is a subgroup of $L_{2 k+1}^{h}(B ; \omega)$. Therefore, the assumption on $x$ implies that $x$ belongs to the kernel of the natural homomorphism

$$
L_{2 k+1}^{h}(G ; \omega \circ \Phi) \rightarrow \Gamma_{2 k+1}^{h}(\mathbf{Z} G \stackrel{\Phi}{\rightarrow} \mathbf{Z} B) .
$$


Hence, by [CS, Proposition 2.1], $(f, b)$ is normally cobordant to $\left(f^{\prime}\right.$, $\left.b^{\prime}\right)$ where $f^{\prime}$ is a $k$-connected $\mathbf{Z} B$-homology equivalence. By Proposition 2.1, $f^{\prime}$ is a rational homology equivalence and then, by [C, Theorem 4.10], $r(x)=0$.

PROOF OF THEOREM 1.

Case 1. $G$ is a finite 2-group. If $\omega$ is the trivial homomorphism, the result follows from Lemma 4.1 applied to $N=G$. If $\omega$ is not trivial, one uses Lemma 4.1 applied to the case $B=\mathrm{Z} / 2 \mathrm{Z}$ and $\Phi=\omega_{G}$ together with the fact that $L_{2 k+1}^{h}(Z / 2 Z ;$ id $)=0$ [W2, Theorem 13.A.1].

Case 2. $G$ is a 2-hyperelementary group. This means that $G$ has a decomposition $1 \rightarrow C \rightarrow G \rightarrow P \rightarrow 1$, where $P$ is a 2-group and $C$ is cyclic. The proof will be by induction on $d(G)$, the greatest odd divisor of $|G|$. If $d(G)=1, G$ is a 2-group and we can use Case 1 . If $d>1$, one may write $C=C_{1} \times C_{2}$, where $C_{1}$ is a cyclic $p$-group with $p \neq 2$, and $\left(p,\left|C_{2}\right|\right)=1$. Thus, one has a split exact sequence:

$$
1 \longrightarrow C_{1} \longrightarrow G \underset{S}{\stackrel{\Phi}{\longrightarrow}} \bar{G} \longrightarrow 1
$$

and $\omega_{G}: G \rightarrow \mathbf{Z} / 2 \mathbf{Z}$ factors through $\omega_{\bar{G}}: \bar{G} \rightarrow \mathbf{Z} / 2 \mathbf{Z} . \bar{G}$ is a 2-hyperelementary group with $d(\bar{G})<d(G)$. By induction hypothesis, $r: L_{2 k+1}^{h}\left(\bar{G} ; \omega_{\bar{G}}\right)$ $\rightarrow L_{2 k+1}^{h}\left(\mathbf{Q} \bar{G} ; \omega_{\bar{G}}\right)$ is zero. Using the functoriality of sequence (3.1), one gets a diagram:

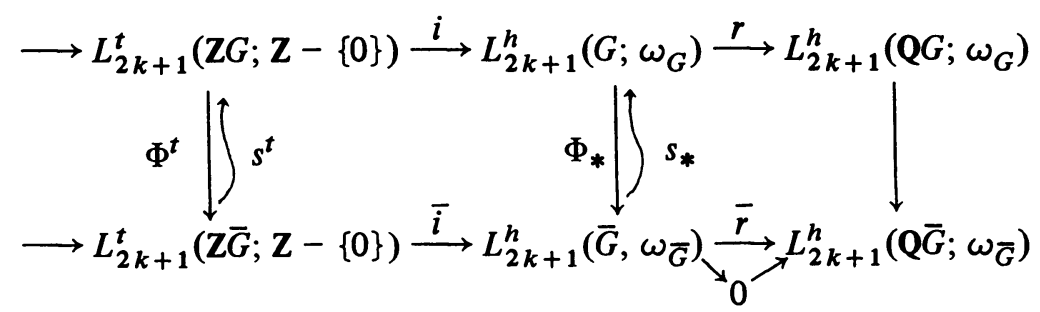

Thus one can write $x \in L_{2 k+1}^{h}\left(G ; \omega_{G}\right)$ as $x=x_{1}+x_{2}$ with $x_{1}$ $=i(y)$ and $x_{2} \in \operatorname{ker} \Phi_{*}$. By Lemma 4.1, $r(x)=0$.

Case 3. General case. Since $L_{\text {odd }}^{h}(G ; \omega)$ is a 2-group [C], the Dress induction theorem [D] asserts that the product of restrictions $L_{2 k+1}^{h}(G ; \omega)$ $\rightarrow \prod_{H \in \mathscr{H}(G)} L_{2 k+1}^{h}(H ; \omega \mid H)$ is injective, where $\mathcal{F}(G)$ is the set of 2-hyperelementary subgroups of $G$. The same holds for $L_{2 k+1}^{h}(Q G, \omega)$. Theorem 1 then follows, using Case 2.

5. Proof of Theorem 3 and Corollaries 4 and 5. Let $M^{2 k}$ be a closed manifold with $\pi_{1}(M)=G$ and orientation character $\omega \circ \Phi$. Represent $y$ as the surgery obstruction for a normal map of degree one:

$$
f:\left(W^{2 k+1}, M, M^{\prime}\right) \rightarrow(M \times I, M \times\{0\}, M \times\{1\}) .
$$

As in the proof of Lemma 4.1, the condition on $y$ implies that $f$ is normally cobordant to an $f^{\prime}$ that is a $k$-connected $\mathbf{Z} B$-homology equivalence. By Proposition 2.1, $f^{\prime}$ is a $\mathbf{Z}_{(2)}$-homology equivalence. Therefore, using [PP, §5], one can consider $y$ as an element of $J_{k}(G)$, the Grothendieck group based on 
linking forms over finite $\mathrm{Z} G$-modules without 2-torsion. $J_{k}(G)$ has exponent 4 [PP, Theorem 5.1]. Thus $4 y=0$.

Corollary 4 is a consequence of Theorem 4 , applied to $N=G$ in the orientable case and to $\Phi=\omega$ otherwise (see the proof of Theorem 1, Case 1). For Corollary 5, one uses Theorem 3 for $N$ equal to the normal 2-Sylow group of $G$, and the fact that $L_{\text {odd }}^{h}(H)=0$ when $H$ has odd order ([Bak] or [P1]).

\section{REFERENCES}

[Bak] A. Bak, Odd dimension surgery groups of odd torsion groups vanish, Topology 14 (1975), 367-374.

[Bak2] - The computation of surgery groups of finite groups with abelian 2-hyperelementary, Algebraic $K$-theory, Lecture Notes in Math., Springer, Berlin and New York, vol. 551, pp. $384-409$.

[Ba] H. Bass, Unitary algebraic K-theory, Proc. Conf. Seattle Res. Center, Batelle Memorial Inst., 1972, Lecture Notes in Math., vol. 343, Springer-Verlag, Berlin and New York, 1973. MR 51 \#8211.

[CS] S. Cappell and J. Shaneson, The codimension two placement problem and homology equivalent manifolds, Ann. of Math. (2) 99 (1974), 277-348. MR 49 \#3978.

[C] F. Connolly, Linking numbers and surgery, Topology 12 (1973), 389-409.

[D] A. Dress, Induction and structure theorem for orthogonal representations of finite groups, Ann. of Math. (2) 102 (1975), 291-326.

[G] R. M. Geist, Semicharacteristic detection of obstructions to rational homotopy equivalence, Notices Amer. Math. Soc. 21 (1974), A-451. Abstract \#74T-G72.

[H] M. Hall, The theory of groups, Macmillan, New York, 1959. MR 21 \# 1996.

[KM] M. Kervaire and J. Milnor, Groups of homotopy spheres. I, Ann. of Math. (2) 77 (1963), 504-537. MR 26 \# 5584.

[P1] W. Pardon, Local surgery and application to the theory of quadratic forms, Bull. Amer. Math. Soc. 82 (1976), 131-134.

[P2] - The exact sequence of a localization for Witt groups, Algebraic $K$-Theory, Lecture Notes in Math., vol. 551, Springer, Berlin and New York, pp. 303-310.

[PP] D. Passman and T. Petrie, Surgery with coefficients in a field, Ann. of Math. (2) 95 (1972), 385-405. MR 46 \# 10011.

[W1] C. T. C. Wall, Surgery of non-simply-connected manifolds, Ann. of Math. (2) 84 (1966), 217-276. MR 35 \#3692.

[W2] - Surgery on compact manifolds, Academic Press, New York, 1970.

[W3] - Classification of hermitian forms. VI. Group rings, Ann. of Math. (2) 103 (1976), $1-80$.

School of Mathematics, Institute for Advanced Study, Princeton, New Jersey 08540

Current address: Section de Mathématiques Université, 2-4 rue de Lièvre, 1211 Geneva 24, Switzerland 